\title{
Cellular Automata Modeling of Continuous Stirred Tank Reactors
}

\author{
J.E. Pérez-Terrazas ${ }^{1 *}$, V. Ibarra-Junquera ${ }^{2 \dagger} \&$ H.C. Rosu ${ }^{1 \ddagger}$ \\ 1 Potosinian Institute of Science and Technology, \\ Apartado Postal 3-74 Tangamanga, 78231 San Luis Potosí, Mexico \\ ${ }^{2}$ Faculty of Chemical Sciences, University of Colima, Coquimatlán, Col., Mexico
}

(Dated: Sept. 24, 2006)

\begin{abstract}
The classical dynamical systems model of continuous stirred tank reactors (CSTR) in which a first order chemical reaction takes place is reformulated in terms of stochastic cellular automata by extending previous works of Seyborg [3] and Neuforth [4] by including the feed flow of chemical reactants. We show that this cellular automata procedure is able to simulate the dilution rate and the mixing process in the CSTR, as well as the details of the heat removal due to the jacket. The cellular automata approach is expected to be of considerable applicability at any industrial scales and especially for any type of microchemical systems.
\end{abstract}

PACS numbers: 82.20.-w 02.70.-c

\footnotetext{
*E-mail: jenrique@ipicyt.edu.mx

$\dagger$ E-mail: vrani@ipicyt.edu.mx

$\ddagger$ E-mail: hcr@ipicyt.edu.mx, Corresponding author
} 


\section{INTRODUCTION}

A chemical reactor could be any vessel containing chemical reactions. In general, a reactor is designed such as to maximize the yield of some particular products while requiring the least amount of money to purchase and operate. Normal operating expenses include energy input, energy removal, raw material costs, labor, etc. Energy changes can occur in the form of heating or cooling, or agitation. The latter is quite important because an appropriate mixing has a large influence on the yield. Therefore, the design and operation of mixing devices often determines the profitability of the whole plant. Theoretically, the effect of stirring in reactant media have also attracted considerable attention [1].

In particular, in the widely developed continuous stirred tank reactors (CSTR) one or more fluid reagents are introduced into a tank equipped with an impeller while the reactor

effluent is removed [2]. The impeller stirs the reagents to ensure proper mixing. Classical CSTR dynamical models, based on coupled deterministic ordinary differential equations (ODEs), are the usual approach to chemical systems at the macroscopic scale. It has been demonstrated to have considerable usefulness. However, their validity relies on many assumptions that limit the situations in which they can be applied. One of the most important is that chemical systems are discrete at the molecular level and statistical fluctuations in concentration and temperature occur at the local scale. The elaboration of models considering this discreteness is important. Seyborg [3] and Neuforth [4] have shown that stochastic cellular automata models can be successfully applied in simulating first order chemical reactions. In their papers, they worked on a squared arrangement of cells, each of them having a chemical reactive. The reactions are performed by considering a probability of change, from reactive $\mathrm{A}$ to reactive $\mathrm{B}$, proportional to the kinetics constant that defines the chemical equation. However, this type of calculation does not apply directly to the CSTR case, where a chemical feed flow is present.

In this paper we extend the stochastic CA model to CSTRs by simulating the feed flow flux by means of a random selection of a subset of cells to which the flux conditions with respect to chemical concentration and temperature are imposed. We would like to remark that mixing in a stirred tank is complicated and not well described despite the extensive usage of dimensionless numbers and models based on ODEs [5]. Therefore, more accurate models are essential for developing and testing control strategies or even to explore new 
reactor geometries. The organization of the paper is the following. Section II presents shortly the standard ODE-based CSTR model. Section III describes the CA method that we implemented for jacketed CSTRs. The simulations are displayed and briefly discussed in Section IV] The paper ends up with several concluding remarks.

\section{THE DETERMINISTIC DYNAMICAL SYSTEMS MODEL}

As already mentioned, we consider an ideal jacketed CSTR where the following exothermic and irreversible first-order reaction is taking place:

$$
A \longrightarrow B
$$

The CSTR modeling equations in dimensionless form are the following [6]

$$
\begin{aligned}
& \frac{d X_{1}}{d \tau}=-\phi X_{1} k\left(X_{2}\right)+q\left(X_{1_{f}}-X_{1}\right) \\
& \frac{d X_{2}}{d \tau}=\beta \phi X_{1} k\left(X_{2}\right)-(q+\delta) X_{2}+\delta X_{3}+q X_{2_{f}} \\
& \frac{d X_{3}}{d \tau}=\frac{q_{c}}{\delta_{1}}\left(X_{3_{f}}-X_{3}\right)+\frac{\delta}{\delta_{1} \delta_{2}}\left(X_{2}-X_{3}\right),
\end{aligned}
$$

where $X_{1}, X_{2}$, and $X_{3}$ are the dimensionless concentration, temperature, and cooling jacket temperature, respectively. We note that it is possible to use the dimensionless coolant flow rate, $q_{c}$, to manipulate $X_{2}$.

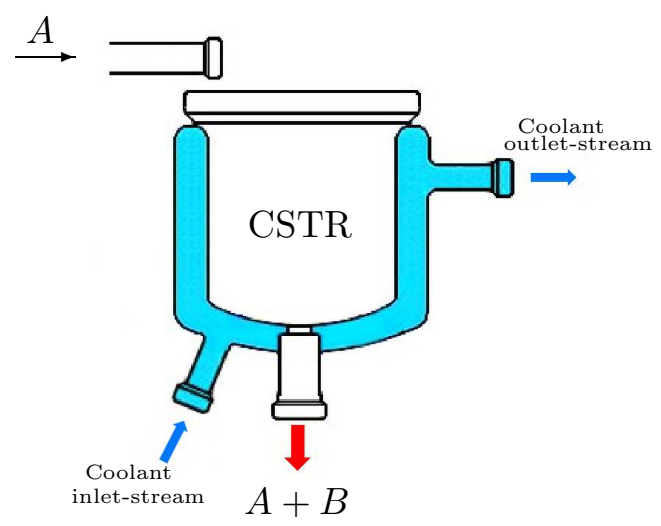

FIG. 1: Schematic representation of the jacketed CSTR.

The relationships between the dimensionless parameters and variables and the physical 
variables are the following:

$$
\begin{gathered}
k\left(X_{2}\right)=\exp \left(\frac{X_{2}}{1+X_{2} \gamma^{-1}}\right), \gamma=\frac{E}{R T_{f_{0}}}, X_{3}=\frac{T_{c}-T_{f_{0}}}{T_{f_{0}}} \gamma, X_{2}=\frac{T-T_{f_{0}}}{T_{f_{0}}} \gamma \\
\beta=\frac{(-\Delta H) C_{f}}{\rho C_{p} t_{f_{0}}}, \delta=\frac{U A}{\rho C_{p} Q_{0}}, \phi=\frac{V}{Q_{0}} k_{0} e^{-r}, X_{1}=\frac{C}{C_{f_{0}}}, X_{1_{f}}=\frac{C_{f}}{C_{f_{0}}} \\
X_{2_{f}}=\frac{T_{f}-T_{f_{0}}}{T_{f_{0}}} \gamma, \delta_{1}=\frac{V_{c}}{V}, \tau=\frac{Q_{0}}{V} t, X_{3_{f}}=\frac{T_{c_{f}}-T_{f_{0}}}{T_{f_{0}}} \gamma, \delta_{2}=\frac{\rho_{c} C_{p_{c}}}{\rho C_{p}}
\end{gathered}
$$

where the meaning of the symbols is given in Table 1 . In the rest of the paper we shall use the solution of this ODE model as the theoretical case with which the CA simulations will be compared.

The nominal parameter values used here are given by:

$$
\begin{array}{r}
\beta=8.0, \delta=0.3, X_{1_{f}}=1.0, X_{3_{f}}=1.0, q=1.0, q_{c_{s}}=1.65 \\
\gamma=20.0, X_{2_{f}}=0.0, \phi=0.072, \delta_{1}=0.1, \delta_{2}=0.5
\end{array}
$$

\section{STOCHASTIC CA MODEL FOR JACKETED CSTR}

The process simulated in this work is the exothermic reaction that converts a chemical A into a product B in a jacketed CSTR. Our model is composed of three squared arrangements of cells, all of the same size. The first lattice is for chemicals A and B and represents the chemical distribution in the tank reactor. In each cell there is only one unit of reactive A or one unit of product $\mathrm{B}$ (not necessary representing a single molecule), under the condition that all cells are occupied. The second arrangement is for the tank temperature. It contains the temperatures $t_{i j}$ in real values, with each cell in this arrangement corresponding to the respective cell in the first arrangement. The third arrangement represents the coolant system. We have used a squared lattice of the same size as the temperature array in such a way that each temperature tank cell is in "contact" with a coolant jacket cell.

In our model the first process in each time step is the irreversible conversion of chemical A into product $\mathrm{B}$. The conversion rate is determined by $\phi k\left(X_{2}\right)$ as in [6], where $X_{2}$ is the average temperature of the tank temperature arrangement. This first order kinetics "constant" is multiplied by the time step in order to get the proportion of the reactive A that is expected to be converted into product B in each evolution step. This number could also be considered as the probability that a molecule of chemical A would be converted in 
TABLE I: Parameters of the model

\begin{tabular}{|c|c|c|}
\hline Symbol & Meaning & \multirow{2}{*}{$\begin{array}{c}\text { Value } \\
\frac{(\text { arb. units) }}{0.001}\end{array}$} \\
\hline$C$ & Reactor composition & \\
\hline$C_{f}$ & Feed composition & 1.0 \\
\hline$q$ & Dimensionless reactor feed flow rate & 1.0 \\
\hline$q_{c}$ & Dimensionless coolant flow rate & 1.65 \\
\hline$q_{c s}$ & Steady-state value of $Q$ & 1.0 \\
\hline$T$ & Reactor temperature & 1.0 \\
\hline$T_{c}$ & Coolant temperature & 1.0 \\
\hline$U A$ & Heat transfer coefficient times the heat transfer area & 1.0 \\
\hline$V$ & Reactor volume & 1.0 \\
\hline$V_{c}$ & Cooling jacket volume & 1.0 \\
\hline$X_{1 f}$ & Dimensionless feed concentration & 1.0 \\
\hline$X_{2 f}$ & Dimensionless feed temperature & 0.0 \\
\hline$X_{3 f}$ & Dimensionless coolant feed temperature & 1.0 \\
\hline$\beta$ & Dimensionless heat of reaction & 8.0 \\
\hline$\delta_{1}$ & Dimensionless volume ratio & 0.1 \\
\hline$\delta_{2}$ & Dimensionless density multiplied by the heat capacity of coolant & 1.0 \\
\hline$\phi$ & Hill's threshold parameter & 0.072 \\
\hline$\gamma$ & Dimensionless activation energy & 20.0 \\
\hline$\rho_{c} C_{p_{c}}$ & Density multiplied by the heat capacity of coolant & 1.0 \\
\hline$\tau$ & Dimensionless time & -- \\
\hline
\end{tabular}

product B if the time step is small enough. Such proportion is compared with a randomly generated number, one different random number for each cell in the arrangement containing reactive $\mathrm{A}$. If the random number is less than the proportion, the reactive $\mathrm{A}$ is changed for product B in the cell. Since the reaction is exothermic, the temperature value in the temperature array is increased by $\beta$ (according to Eq. (2)) in the corresponding cell. 
The second simulated process in our model is the tank temperature diffusion that can be simultaneously considered as an energy diffusion. It can be performed by means of finite differences, but in order to obtain a model almost ODE independent we have implemented a moving average method, where the value of the temperature in a cell at the next time step is the average temperature of its neighborhood. This procedure gives similar results to those of finite differences, as shown by Weimar for reaction-diffusion systems simulated by cellular automata [7]. We used a square neighborhood formed by $(2 R+1)^{2}$ cells, where $R$ is the number of steps that we have to walk from the center of the cell in order to reach the most far horizontal (vertical) cell in the neighborhood.

The third simulated process is the tank feed flow. We have simulated the feed flow rate $q$ in a stochastic way. In order to get an approximation to the proportion of the tank that must be replaced by the incoming flow, $q$ is multiplied by the time step and by the total number of cells in the arrangement. This give us a real number $x$. Then, following Weimar [7], we used a probabilistic minimal noise rule, i.e. it is defined the probability $p=x-\lfloor x\rfloor$ in order to decide if $\lfloor x\rfloor$ or $\lfloor x\rfloor+1$ cells will be replaced by the flow. We choose $\lfloor x\rfloor$ with probability $1-p$ and $\lfloor x\rfloor+1$ with probability $p$. This method conserves the proportion $x$ in a statistical way. Subsequently, a cell is selected in a random way, by means of two random numbers which are used to select a row and a column, in such a way that all cells has the same probability of being selected. If the cell has been selected in the same time step, a new selection is made. This is repeated until we have reached the number of cells that must be replaced. Finally, the selected cells are changed in the temperature arrangement by the feed flow temperature $X_{2 f}$, and in the reactive arrangement it is put a unit of reactive A with probability $X_{1 f}$, that represent the concentration of chemical A in the feed flow. In the simulations presented in this work we used $X_{1 f}=1$. This method of flow simulation could be improved in several ways, in order to simulate different tank geometries or for showing the flow direction. However, in this work we want only to show that the CA method could fit the CSTR behavior in a very good approximation, with the advantage of spatial analysis.

The fourth simulated process is the energy interchange between the tank and the jacket. This has been done by directly calculating the energy/temperature interchange between each tank temperature cell and its corresponding jacket temperature cell. This interchange is dictated by the difference between the two temperatures and it is weighted by $\delta, \delta_{1}$, and 
$\delta_{2}$ as in Eqs. (1) and (2). The fifth and the sixth simulated processes are the coolant flow and the coolant temperature diffusion, respectively. Both of them are performed in a similar way as for the concentration and temperature tank.

\section{SIMULATIONS}

In this section we first present the comparison between the curves obtained by differential equations and those obtained from the implemented cellular automata model (see Fig. 2). It is clear that cellular automata simulations resemble with excellent agreement the values for the concentration of chemical A, the tank temperature and the jacket temperature at all times. Using a time step of 0.001 it is shown that the curves coincide at the initial time, transient time and for stable state. We have found that we can maintain this remarkable fitness by properly adjusting the time step to a sufficiently small value.

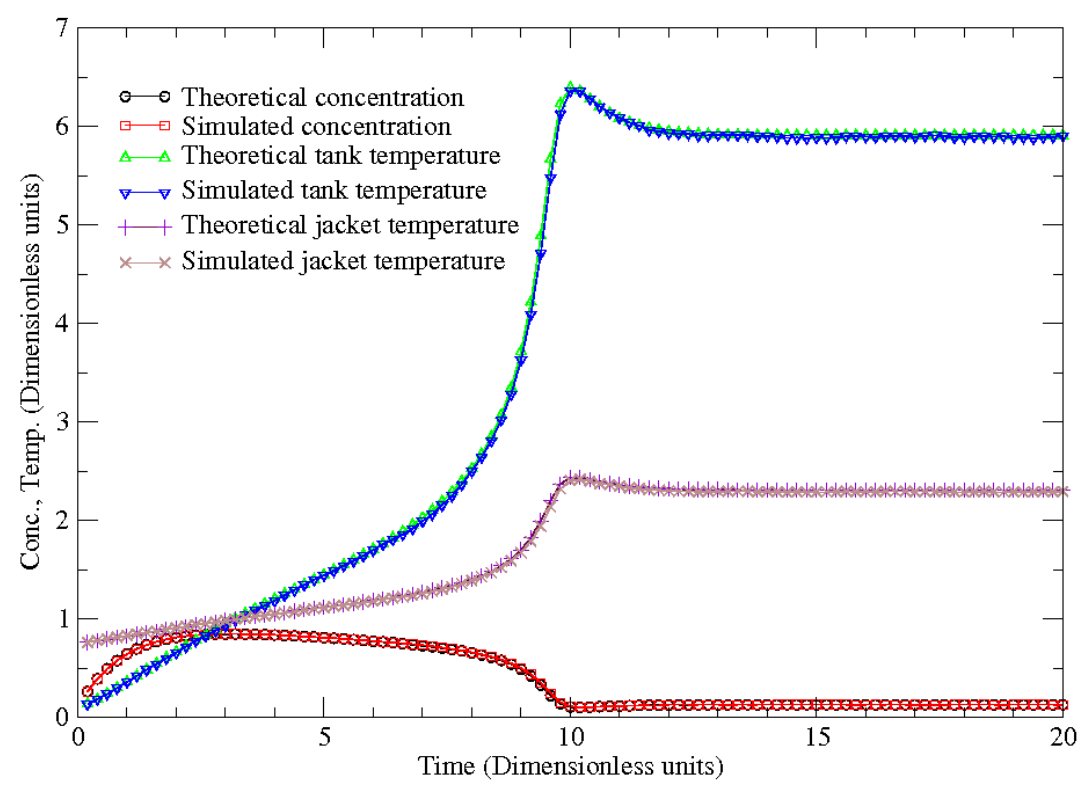

FIG. 2: Comparison between the curves from the differential equations and the curves obtained from simulations with the cellular automata approach. Initial values are: $X_{1}=0.1, X_{2}=0.1$, $X_{3}=0.1$. For the six curves: 100 points separated by a dimensionless time of 0.2 were taken from 20000-point simulations with a dimensionless time step of 0.001 . 
When a kinetic constant based on the average temperature is used, it is implicity assumed that the mixing in the CSTR is perfect, vanishing any temperature inhomogeneities. One could ask what could be the change in the tank behavior if the mixing is almost perfect. It could be studied by using a model that consider the spatial distribution of temperature. We studied this effect by calculating the kinetic constant for each cell based in the its correspondent temperature. The effect for a $1000 \times 1000$ cells array and a time step of 0.001 is shown in Fig 3. It could be observed that the tank temperature curve for the perfect mixing and the one for the locally calculated kinetic constant are the same for almost all times. However, they separate during the transient period, leading to a reduction in the magnitude of the peak and a little delay in its appearance. The curve was calculated with a tank temperature diffusion process per time step with a $R=1$ neighborhood. If more diffusion steps are used per time step the curves obtained tend to the theoretical one as is expected. Besides, it could be also interpreted as the effect of a perfect mixed tank, but with material where each component tends to maintain its energy.

The ODEs generally represent the characteristics of the global system, where it has enough number of elements such that the statistical fluctuations are of small amplitude. However, when the system size and the quantity of elements are diminished, the statistical fluctuations could be of increasing importance. In this way, another advantages of the cellular automata method proposed here is its flexibility with respect to the reactor size, and its stochastic nature, that allows to study how much the system could be affected by the initial conditions and by the stochastic features of the process.

We have performed several simulations applying the parameters presented above in arrangements of small size, where the model is no longer representative for a 3-dimensional CSTR at such scales. However, we can think of it as a representation of a catalytic surface dividing two regions, one carrying the chemical A and the other as a temperature reservoir. Therefore, this model is a simple approach, useful as a first approximation, in the analysis and study of microreactors or even nanoreactors. We recall that the usage of microreactors for in situ and on-demand chemical production is gaining increasing importance as the field of microreaction engineering has already demonstrated potential to impact a wide spectrum of chemical, biological, and process system applications [8]. There are already many success- 


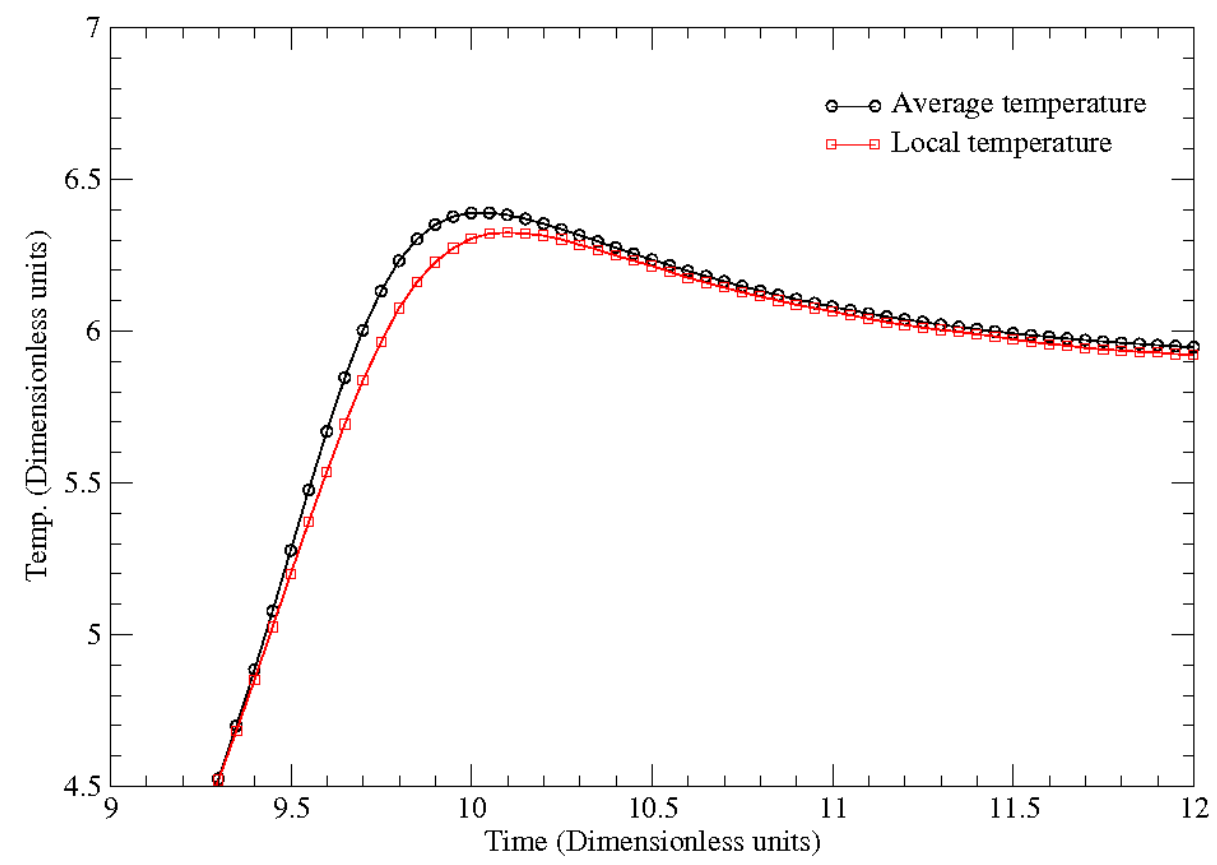

FIG. 3: Comparison between the tank temperature evolution curves for a kinetic constant based on average temperature and for kinetics constant calculated on the base of the local temperature. Initial values are: $X_{1}=0.1, X_{2}=0.1, X_{3}=0.1$. The array is of $1000 \times 1000$ cells and the dimensionless time step is of 0.001 ; one temperature diffusion step per time step.

fully developed microreactors for chemical applications such as partial oxidation reactions 9], phosgene synthesis [10], multiphase processing [11], and (bio)chemical detection [12].

Figure 4 displays the variability that could be found in CSTR systems at small scales. It is clear evidence that the statistical fluctuations are a primordial issue at this scale. In addition, one could notice that the dynamical behavior could be totally different to the expected behavior of a larger system e.g., $1000 \times 1000$ cells arrangement.

Finally, the study of small systems by direct simulation using stochastic simulations could give us insight in how an open CSTR system could behave when the statistical fluctuations and the initial configuration are important. In Fig. 5 one can see that the possible behaviors of a system of size $20 \times 20$ have big deviations from the average (theoretical) value. This kind of variability is not provided by pure ODEs (without a stochastic term). We think 


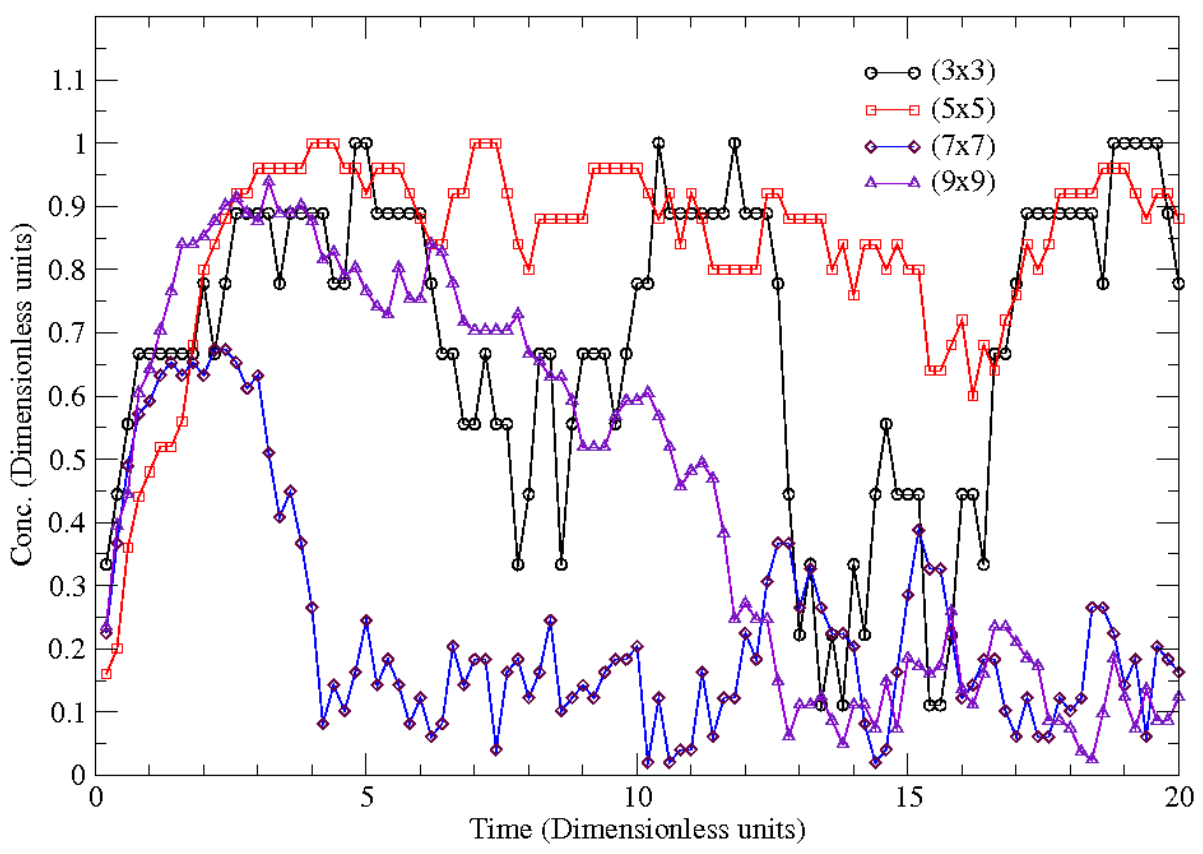

FIG. 4: Behaviors that could be found in systems with small number of elements (cells for the CAs and clusters of molecules in the real case). These behaviors are different from that expected in systems with a large number of elements. The initial values are $X_{1}=0.1, X_{2}=0.1, X_{3}=0.1$. The employed time step is 0.001 . One hundred points separated by a time lag of 0.2 were taken from 20000-point simulations with a time step of 0.001 ; one temperature diffusion step per time step.

that this stochastic CA approach could be an important tool for testing control strategies since the CA approach could be seen as a step between the ODEs models and the specific experimental situation.

\section{CONCLUDING REMARKS}

A cellular automata approach for the CSTR with cooling jacket has been presented in this paper. It is able to reproduce the CSTR dynamical behavior calculated by ODE's with a good approximation and in an easy way. The stochastic model presented allow us to 


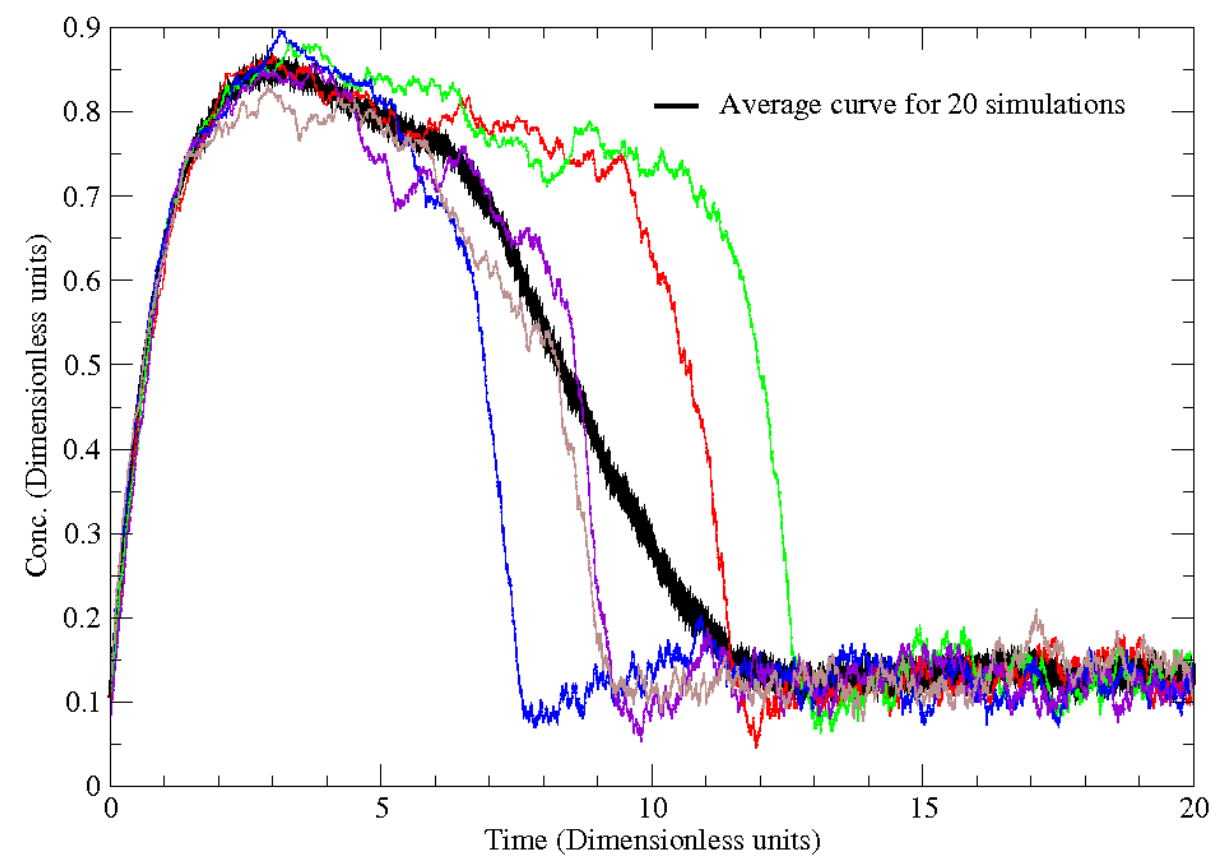

FIG. 5: Different concentration behaviors of chemical A for systems of the same size $(20 \times 20)$ that are treated by the same method and could be the underlying dynamic characteristic of microreactors. Five simulations are displayed together with the average curve (thick line) of 20 individual simulations. The initial configuration and the stochasticity introduced in the model lead to a timedistributed behavior. The initial values are: $X_{1}=0.1, X_{2}=0.1, X_{3}=0.1$. The time step is 0.001 ; one temperature diffusion step per time step.

study what could be the behavior of the variables of the tank when the reaction probability depends on the local temperature. It also give us an approach to study systems with few elements, as could be micro and nanoreactors, as could be catalytic membranes separating two phases. The main advantages of the CA approach presented here are its stochastic nature and the direct involvement of a spatial structure. This also represents a tool for studying the role of initial configuration and stochastic fluctuations in systems with few elements. Additionally, the CA approach is a clear improvement of the CSTR modeling and moreover can be applied to different reactor and jacket geometries, as well as for considering 
in more detail the real mass flow in the tank reactor-geometry.

[1] R. Reigada, F. Sagués, I.M. Sokolov, J.M. Sancho, and A. Blumen, Phys. Rev. Lett. 78, 741 (1997); D.H. Zanette, Phys. Rev. E 52, 1213 (1995); ibid. 50, 1171 (1994); A. Puhl, V. Altares, and G. Nicolis, Phys. Rev A 37, 3039 (1988).

[2] I.R. Epstein and J.A. Pojman, An Introduction to Nonlinear Chemical Dynamics (Oxford University Press, Oxford, 1998).

[3] P.G. Seybold, L.B. Kier, and C.-K. Cheng C.-K., J. Chem. Inf. Comput. Sci. 37, 386 (1997).

[4] A. Neuforth, P.G. Seybold, L.B. Kier, ands C.-K. Cheng, Int. J. Chem. Kinet. 32, 529 (2000).

[5] S. Chakraborty and V. Balakotaiah, Chem. Eng. Sci. 58, 1053 (2003).

[6] R.G. Silva and W.H. Kwong, Braz. J. Chem. Eng. 16, 83 (1999).

[7] J. R. Weimar, Parallel Computing 23, 1699 (1997).

[8] K.F. Jensen, Chem. Eng. Sci. 56, 293 (2001).

[9] I.M. Hsing, R. Srinivasan, K.F. Jensen, M.A. Schmidt, Chem. Eng. Sci. 55, 3 (2000).

[10] S.K. Ajmera, M.W. Losey, and K.F. Jensen, AIChE J. 47, 1639 (2001).

[11] N. de Mas, A. Günther, M.A. Schmidt, and K.F. Jensen, Ind. Eng. Chem. Res. 42, 698 (2003).

[12] W.-G. Koh and M. Pishko, Sensors and Actuators B: Chemical 106, 335 (2005). 